Bioscientia Medicina: Journal of Biomedicine \& Translational Research

Journal Homepage: www.bioscmed.com

\title{
Correlation Between Candidiasis and Mortality in Gastric Ulcer Perforation Patients
}

\section{Faradina Sulistiyani ${ }^{1 *}$, Vicky Sumarki Budipramana², Pepy Dwi Endraswari ${ }^{3}$}

${ }^{1}$ General Surgery Department, Airlangga Medical Faculty, Soetomo General Hospital, Surabaya, Indonesia

2 Digestive Surgery Department, Airlangga Medical Faculty, Soetomo General Hospital Surabaya, Indonesia

${ }^{3}$ Microbiology Department, Airlangga Medical Faculty, Soetomo General Hospital Surabaya, Indonesia

\section{A R T I C L E I N F O}

\section{Keywords:}

Candidiasis

Gastric ulcer perforation

Mortality

\section{*Corresponding author:}

Galih Aktama

\section{E-mail address: \\ faradinaSulistiyani@gmail.com}

All authors have reviewed and approved the final version of the manuscript.

\begin{abstract}
A B S T R A C T
Background: Gastric ulcer disease remains the most common gastrointestinal disease. This affects about 4 million people every year worldwide with an incidence of $1.5 \%-3 \%$ and $2-14 \%$ of these will progress to perforation. Mortality is reported in $30 \%$ of cases and caused morbidity in $50 \%$ of patients. Some studies have suggested that mortality in patients with peritonitis accompanied by candidiasis ranges from $20 \%-75 \%$, whereas others have argued that the presence of fungal isolates in patients with perforated peptic ulcer does not affect the outcome. A recent study reported an incidence of $45 \%$ of patients peptic ulcer perforation cases accompanied by candidiasis in Dr. Soetomo General Hospital Surabaya in 2019. Methods: This was a cross sectional study based on medical record data of patients with gastric ulcer perforation accompanied by candidiasis who underwent laparotomy and gastric repair surgery at Dr. Soetomo General Hospital Surabaya from January 2019 to December 2020. Results: There was no significant correlation between candidiasis and mortality in gastric ulcer perforation patients $(p=0.989)$. Conclusion: Candidiasis does not increase mortality in patients with gastric ulcer perforation.
\end{abstract}

https://doi.org/10.32539/bsm.v5i11.370

\section{Introduction}

Peptic ulcer disease remains the most common and expensive gastrointestinal disease. ${ }^{1}$ Nearly 4 million people worldwide are diagnosed with peptic ulcer each year. The incidence of peptic ulcer is estimated to be around 1, 5\%-3\%. Complications occur in 10-20\% of patients, while $2-14 \%$ of patients will experience perforation. ${ }^{2}$ Peptic ulcer perforation is a common surgical emergency with a prevalence of around 5\% and is associated with mortality of up to $30 \%$ and morbidity of up to $50 \% .^{3}$ The mortality rate of gastric perforation patients with a Boey score of 2 at dr. Soetomo Hospital Surabaya in 2019 was reported to be $53 \%$.

The most common causes of gastric perforation include taking NSAIDs. ${ }^{4}$ Gastric perforation can also be accompanied by fungal infection and is associated with a significant increase in morbidity and mortality. ${ }^{1}$ It has been reported that mortality in patients with peritonitis accompanied by Candida sp. ranged from $20 \%-75 \% .{ }^{5}$ Meanwhile, the number of cases of gastric perforation accompanied by Candida sp. in Dr. Soetomo Surabaya Hospital in 2019 was reported to have occurred in $45 \%$ of patients.

Studies reported that patients with positive fungal cultures had a higher incidence of surgical site infection, residual abscess formation, longer ICU and hospital stays and higher mortality rates than patients with negative fungal cultures and the results were statistically significant. ${ }^{6}$ However, it has also been 
reported that there were no differences in outcome and the presence of fungal infections in these patients. ${ }^{7}$ Because of conflicting reports, more evidence is needed. Our study aimed to prove the correlation between candidiasis and mortality in gastric ulcer perforation who underwent laparotomy and gastric repair surgery at Dr. Soetomo General Hospital Surabaya from January 2019 to December 2020.

\section{Methods}

This was a cross sectional study based on medical record data of gastric ulcer perforation patients with Boey score 2 at Dr. Soetomo General Hospital Surabaya from January 2019 to December 2020. The patients included in the study were all the patients operated for gastric ulcer perforation, whose microbiological examination results from the biopsy edge of the perforation showed Candida sp. The variables in this study used data with nominal characters so that a chisquare analysis was performed.

\section{Result}

In this study, there were 44 research subjects with the following descriptions: 4 subjects aged 41-50 years (9.1\%), 14 subjects $51-60$ years (31.8\%) and $59.1 \%$ subjects aged 61-70 years old. A total of 21 of 44 patients (47.7\%) had candidiasis. The description of the research subject can be seen in table 1 .

Table 1. Characteristics of Subjects

\begin{tabular}{|c|c|c|}
\hline \multicolumn{2}{|c|}{ Characteristics } & Details \\
\hline \multicolumn{2}{|l|}{ Age } & \\
\hline \multicolumn{2}{|l|}{ Range } & $41-70$ \\
\hline \multirow{2}{*}{\multicolumn{2}{|c|}{$\begin{array}{l}\text { Mean } \pm \\
\text { SD }\end{array}$}} & 61.5 \\
\hline & & 7.708 \\
\hline \multicolumn{2}{|c|}{ Age category } & \\
\hline \multicolumn{2}{|c|}{ 41-50 y.o. } & 4 (9.1\%) \\
\hline \multicolumn{2}{|l|}{ 51-60 у.о. } & 14 (31.8\%) \\
\hline \multicolumn{2}{|l|}{ 61-70 у.о. } & $26(59.1 \%)$ \\
\hline \multirow[t]{2}{*}{ Candida } & Positive & $21(47.7 \%)$ \\
\hline & Negative & $23(52.3 \%)$ \\
\hline \multirow[t]{2}{*}{ Outcome } & Die & $23(52.3 \%)$ \\
\hline & Life & $21(47.7 \%)$ \\
\hline
\end{tabular}

Analysis showed that there was no significant correlation between gender $(p=0.892)$, age $(p=0.634)$, shock $(\mathrm{p}=0.599)$ and comorbidities in the form of hypertension $(p=0.537)$, diabetes mellitus $(p=1.000)$ and pneumonia $(\mathrm{p}=1.000)$ among patients with or without candidiasis (Table 2). While in Table 3, the analysis showed no significant correlation between characteristics and mortality in patients with gastric perforation.

Table 2. Analysis of Characteristics with Candidiasis

\begin{tabular}{|c|l|c|c|c|}
\hline \multicolumn{2}{|c|}{ Characteristics } & \multicolumn{2}{c|}{ Candida } & \multirow{2}{*}{ p-value } \\
\cline { 2 - 4 } & $41-50$ y.o. & $1(4.8 \%)$ & $3(13.0 \%)$ & \\
\hline \multirow{3}{*}{ Age } & $51-60$ y.o. & $7(33.3 \%)$ & $7(30.4 \%)$ & \multirow{2}{*}{0.634} \\
\cline { 2 - 4 } & $61-70$ y.o. & $13(61.9 \%)$ & $13(56.5 \%)$ & \\
\hline \multirow{2}{*}{ Shock } & Yes & $7(33.3 \%)$ & $6(26.1 \%)$ & \multirow{2}{*}{0.599} \\
\cline { 2 - 5 } & No & $14(66.7 \%)$ & $17(73.9 \%)$ & \\
\hline \multirow{3}{*}{ Comorbid } & HT & $9(52.2 \%)$ & $12(52.4 \%)$ & 0.537 \\
\cline { 2 - 5 } & DM & $4(19.0 \%)$ & $5(21.7 \%)$ & 1.000 \\
\cline { 2 - 4 } & Pneumonia & $2(9.5 \%)$ & $3(13.0 \%)$ & 1.000 \\
\hline
\end{tabular}

Note: Chi-Square test is significant p-value $<0.05$ 
Table 3. Analysis of Characteristics with Mortality

\begin{tabular}{|c|c|c|c|c|}
\hline \multirow{2}{*}{\multicolumn{2}{|c|}{ Characteristics }} & \multicolumn{2}{|c|}{ Candida } & \multirow{2}{*}{$p$-value } \\
\hline & & Positive & Negative & \\
\hline \multirow{3}{*}{ Age } & 41-50 y.o. & $2(8.7 \%)$ & $2(9.5 \%)$ & \multirow{3}{*}{0.907} \\
\hline & 51-60 y.o. & $8(34.8 \%)$ & $6(28.6 \%)$ & \\
\hline & 61-70 у.о. & $13(56.5 \%)$ & $13(61.9 \%)$ & \\
\hline \multirow[t]{2}{*}{ Shock } & Yes & 9 (39.1\%) & $4(19.0 \%)$ & \multirow{2}{*}{0.145} \\
\hline & No & 12 (60.9\%) & $19(81.0 \%)$ & \\
\hline \multirow{3}{*}{ Comorbid } & HT & $10(43.5 \%)$ & $11(52.4 \%)$ & 0.555 \\
\hline & $\mathrm{DM}$ & $6(26.1 \%)$ & $3(14.1 \%)$ & 0.462 \\
\hline & Pneumonia & 1 (4.3\%) & 4 (19.0\%) & 0.176 \\
\hline
\end{tabular}

Note: Chi-Square test is significant $p$-value $<0.05$

Table 4 shows that most of the patients who died $52.2 \%$ (12 of 44 patients) were from the group without candidiasis. The results of the analysis also show that statistically, there was no significant correlation $(\mathrm{p}=0.989)$ between candidiasis and mortality in gastric perforation patients $(\mathrm{OR}=1.008 ; 95 \% \mathrm{CI}$ : 0.308-3.296).

Table 4. Analysis of Candidiasis with Mortality

\begin{tabular}{|l|c|c|c|c|c|}
\hline \multirow{2}{*}{ Characteristic } & \multicolumn{2}{|c|}{ Mortality } & P-value & OR (CI 95\%) \\
\cline { 2 - 5 } & Die & $10(47.6 \%)$ & \multirow{2}{*}{0.989} & $1.008(0.308-3.296)$ \\
\hline \multirow{2}{*}{ Candida } & $\mathbf{( + )}$ & $11(47.8 \%)$ & $11(52.4 \%)$ & & \\
\cline { 2 - 5 } & $\mathbf{( - )}$ & $12(52.2 \%)$ & & \\
\hline
\end{tabular}

\section{Discussion}

In this study, we evaluated mortality in gastric perforation patients with candidiasis. Specifically, there were $21 / 44$ (47.7\%) gastric perforation patients with candidiasis based on microbiological examination of the tissue. The incidence of intestinal mycoses was reported by Eras et al., who found 109 (4.35\%) of the total 2517 cases studied from 1960-1964.8 In 1982, Minoli et al. reported that gastric candidiasis occurred in $0.96 \%$ of 2700 patients who underwent endoscopy from 1978-1980.9 Values attained in this study are significantly higher in comparison to previous study published by Lee et al. (2002), which found $37.1 \%$ of patients with perforated peptic ulcer with candidiasis. The study by Jindal et al. (2015) also recorded 68 patients with gastrointestinal perforation (48.6\%) out of a total of 140 patients experiencing candidiasis. Available evidence supports higher incidence of candidiasis in gastrointestinal perforation patients.

In this study, the highest incidence of gastric perforation was found in the age group 61-70 years and in the group with candidiasis compared to those without candidiasis $(61.9 \%$ vs $56.5 \%)$. This is in accordance with the previous theory which states that gastric perforation is rare under 40 years of age, with a peak incidence at 55-65 years of age. ${ }^{10}$ It has also been reported that age more than 60 years was the main risk factor affecting outcome, postoperative mortality in the elderly was 3 to 5 times higher. ${ }^{2}$ Patients older than 65 years were associated with a higher mortality rate when compared to younger patients $(37.7 \%$ vs $1.4 \%)$. This may be due to comorbidities, delayed onset of symptoms, or delay in diagnosis. 
The higher incidence of candidiasis in this study is thought to be due to the wider use of corticosteroids and NSAIDs that can be obtained either by prescription due to comorbidities in elderly patients, or freely obtained without a prescription. This is in line with the study by Agustin et al. (2016) who reported that 66 patients with gastric perforation, $30.3 \%$ of them had a history of long-term NSAID consumption and 53.03\% had a history of consuming traditional herbal medicine.

It is difficult to determine whether intestinal candidiasis is idiopathic or secondary, because Candida species are part of the normal gut flora in healthy people. Under normal circumstances Candida levels are controlled by beneficial bacteria. However, if the bacteria-fungal balance is disturbed by the use of antibiotics or if the immune system is compromised, an overgrowth of Candida can lead to infection. There are several theories on the underlying mechanism of the gastric mucous membrane disruption that was sufficient to cause gastric candidiasis. Some studies have reported that several cases of idiopathic gastric candidiasis in which no underlying disease was present, whereas others have reported that Candida secondarily invaded and multiplied at the base of the ulcer. ${ }^{4}$

In line with the initial study by Boey et al. (1982), we report that patients with 2 factors who underwent laparotomy had a 52,3\% mortality rate. ${ }^{11}$ The results of this study indicate that the mortality rate on Boey score 2 who underwent laparotomy is still high, so studies on factors that can affect mortality are needed. Variables such as age $(p=0.907)$, septic shock $(p=0.145)$ and comorbidities which consist of hypertension $(p=0.555)$, diabetes mellitus $(p=0.462)$ and pneumonia $(p=0.176)$ did not show any significant correlation with mortality.

The results of this study show no significant correlation $(\mathrm{p}=0.989)$ between candidiasis and mortality in patients with gastric perforation $(\mathrm{OR}=$ 1.008; 95\%CI: 0.308-3.296). These findings are in line with results reported by other publications in literature. ${ }^{7}$ The generally accepted theory is that because Candida sp. is part of the normal intestinal flora in healthy individuals and candidiasis in these patients belongs to community-acquired fungal infection so that adequate source control is expected to be sufficient to treat patients.

As a commensal organism, at first Candida sp. will attach to host cells through yeast forms that live normally on the mucosal surface without causing an immune response. Candida sp. When in the form of hyphae, they can invade and infiltrate the tissue. Hyphae can also invade blood vessels in the digestive tract. Invasive procedures that disrupt the mucosal surface, such as surgery for gastric perforation and immunosuppression, facilitate local invasion and further candidaemia. Penetration of Candida sp. to the mucosa will activate mediators including activation of the complement cascade that initiates opsonization, phagocytosis and intracellular damage of these fungi. Immune defense, especially involving cellular components, is mainly carried out through the process of phagocytosis and induce various immune processes. Infection with Candida activates complement and inflammatory processes, and recruits and activates effector cells such as macrophages, neutrophils, eosinophils, basophils, mast cells and other inflammatory cells. Neutrophils are the most important immune effector cells in the phagocytosis of fungi. Some proinflammatory cytokines will also induce activation of plasma cells (effector cells) to secrete immunoglobulins on their cell surfaces to become antibodies. The main antibody that plays a role in fungal infections is $\operatorname{IgE}$. IgE will induce an immediate type hypersensitivity reaction (allergy). This process will induce degranulation of mediators from effector cells which will then induce tissue damage. During the study period, the average hospitalization for patients with candidiasis was 10 days. This is thought to be because new antibodies are formed after 10 days, so if the patient dies in less than 10 days it may be due to the absence of an immune role. While the immune response has not been formed, the patient also does not receive antifungal intervention so that the treatment takes longer.

In our study, it appears that bacterial and fungal cultures and antimicrobial sensitivity of the specimens are important for the treatment of perforated peritonitis patients. Although there is no significant correlation 
between candidiasis and the mortality of patients with gastric perforation, the high incidence of candidiasis is one of the risk factors for patients. Efforts aimed at timely source control of infection and antifungal treatment are likely to be associated with better clinical outcomes.

\section{Conclusion}

Candidiasis does not increase mortality in patients with gastric perforation.

\section{Ethical Clearance}

This research has received ethical clearance approval from the ethics committee of Dr. Soetomo Hospital.

\section{Conflict of Interest}

This research has no conflict of interest or affiliation with any company.

\section{Funding}

This research did not use sponsorship.

\section{Author Contributions}

Author contributed in conceptualizing, research design, data collection, data processing and this publication.

\section{References}

1. Singla, S., Meena, K., Pathania, O., Agarwal, S., Dev, N., \& Jha, S. (2018). a Common Pathology, a Rare Cause - a Case Report of Candidial Gastric Perforation. International Journal of Surgery and Medicine, 3(3), 1. https://doi.org/10.5455/ijsm.candidagastric-perforation

2. Chung, KT, \& Shelat, VG (2017). Perforated peptic ulcer - an update. 9(1), 112.https://doi.org/10.4240/wjgs.v9.i1.1

3. Søreide, K., Thorsen, K., Harrison, EM, Bingener, J., Møller, MH, Ohene-Yeboah, M., \& Søreide, JA (2015). Perforated peptic ulcer. The Lancet, 386(10000), 1288-1298. https://doi.org/10.1016/S01406736(15)00276-7

4. Md. Mukhtar Naved, Nishant, Asim A. Minj, Shital Malua. A rare case of gastric perforation by candida albicans: a case report. International Journal of Contemporary Medical Research 2017;4(1):76-77.

5. Zhan, J., Shu, G., Yuan, L., Zhu, J., \& Xie, B. (2015). Clinical Features of Fungal Peritonitis with Candida Albicans Infection after Gastric and Duodenal Perforation Emergency Medicine : Open Access. 5(4), 6-8. https://doi.org/10.4172/2165-7548.1000264

6. Jindal N, Arora S, Pathania S. Fungal Culture Positivity in Patients with Perforation Peritonitis.J Clin Diagn Res. 2015;9(6):DC01DC3. doi:10.7860/JCDR/2015/13189.6050

7. Horn, CB, Coleoglou Centeno, AA, Rasane, RK, Aldana, JA, Fiore, NB, Zhang, Q., Torres, M., Mazuski, JE, Divine, ON, Punch, LJ, \& Bochicchio, GV (2018) . Pre-Operative AntiFungal Therapy Does Not Improve Outcomes in Perforated Peptic Ulcers. Surgical Infections, 19(6), 587-592. https: / / doi.org/10.1089/sur.2018.058

8. Eras, P., Goldstein, MJ, \& Sherlock, P. (1972). Candida Infection Of The Gastrointestinal Tract. Medicine, 51(5), 367-79. DOI:10.1097/00005792-197209000-00002

9. Minoli, G., Terruzzi, V., Butti, G., Frigerio, G., \& Rossini, A. (1982). Gastric candidiasis: an endoscopic and histological study in 26 patients. Gastrointestinal Endoscopy, 28(2), 59-61.https://doi.org/10.1016/S00165107(82)72998-0

10. Koto, K. (2016). Characteristic of gastric perforation type and the histopathology at Haji Adam Malik general hospital Medan-Indonesia. $5(1)$, 186-188. https://doi.org/10.15562/bmj.v5i1.325

11. Boey, J. and Wong, J. (1982) 'A prospective study of operative risk factors in perforated duodenal ulcers', Annals of Surgery, 195(3), pp. 265-269. doi:10.1097/00000658198203000-00004. 